\title{
Modeling and Optimization of Capacitive Converter for Energy Scavenging System
}

\author{
Jinxin Huang, Nannan Gao, Hongbo Li \\ State Grid of China Technology College, Jinan, China \\ Email: linbowhjx@163.com
}

Received March, 2013

\begin{abstract}
A new converter with spherical cap for energy scavenging is proposed. Based on the method of separated variables within the torrid coordinate system, a corresponding analytical model for spherical cap converter is further established so as to obtain the analytic expressions of the topology capacitance and the output voltage. The concept of energy increment factor is specifically defined to denote the improvement of energy storage efficiency. With regard to spherical cap converters of different dimensions, the measured values of energy increment factor coincide well with the theoretical equivalents, indicating an effective verification of the proposed analytical model for the spherical cap converter topology.
\end{abstract}

Keywords: Energy Scavenging System; Capacitive Converter; Spherical Cap Topology; Energy Increment Factor

\section{Introduction}

With the development of smart grid and wireless sensor technology, it's necessary to study energy scavenging technology [1-5] to collect the energy in the surrounding environment (such as solar energy, vibration energy, thermal energy and electromagnetic energy) and convert them into electricity for powering sensor nodes. There contains much electromagnetic energy in high voltage substations and it provides a basis for energy scavenging system based on electric field energy. Under the specific environmental conditions in substations, the system has some unique advantages compared with other ones.

At present, a British research team has conducted a preliminary study of it and this provides a fundament for reference [6]. A traditional flat-plate capacitive converter is adopted in their researches. The electric field is very complex in the substations, so the electric field surrounding the capacitive converter maybe come from different directions. For the traditional flat-plate one, it has good effect only at the vertical direction. In order to collect the electric field energy preferably from different directions, a new capacitive topology with spherical cap is proposed, as Figure $\mathbf{1}$ shows. This new topology is not only more suitable for collecting all directions of the electric field, but also conducive to offer electromagnetic shielding for conditioning unit, and avoid marginal discharge.

It can increase energy storage capacity of the converter by optimizing the parameters. This depends on the accu- rate modeling and analysis of the converter. The opening of converter destroys the spherical symmetry, so it becomes difficult to solve the charge and electric potential distribution and capacitance expressions directly in the Cartesian coordinate system. Calculation model of the converter in the torrid coordinate system [7] is established to get analytic formulas of non-complete spherical cap by using variables separation and Laplace equation.

\section{Mathematical Modeling of Capacitive Converter}

According to different sizes of spherical topology, it can be divided into three cases: more halfsphere, halfsphere, and less half sphere which corresponding $\delta$ and $\beta_{0}$ are different, as shown in Figure 2. Torrid coordinate system is a generalized orthogonal curvilinear coordinates, the three parameters are $(\alpha, \beta, \varphi)$ and they have a relation with Cartesian coordinates $(x, y, z)$ as equation (1) shows.

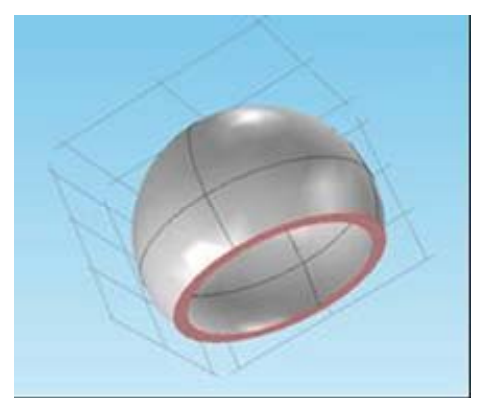

Figure 1. Capacitive converter with spherical cap. 


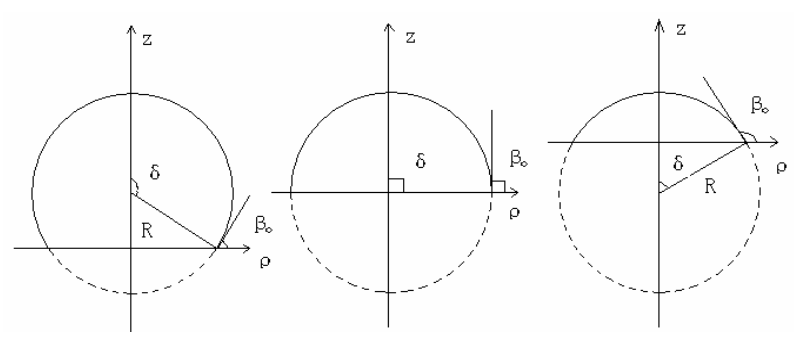

Figure 2. Analytical model of spherical cap converter in torrid coordinate system.

$$
\left\{\begin{array}{l}
x=\frac{a \operatorname{sh} \alpha \cos \varphi}{\operatorname{ch} \alpha-\cos \beta} \\
y=\frac{a \operatorname{sh} \alpha \sin \varphi}{\operatorname{ch} \alpha-\cos \beta} \\
z=\frac{a \sin \beta}{\operatorname{ch} \alpha-\cos \beta}
\end{array}\right.
$$

where, $0 \leq \alpha<\infty, 0 \leq \beta \leq 2 \pi, \quad 0 \leq \varphi<2 \pi, a$ is a parameter in torrid coordinate and denotes opening radius of the spherical cap.

Assume that the radius is $R$, the electric potential is $u$. External space of the converter meets the Laplace equation $\nabla^{2} u=0$. In torrid coordinate system, $\varphi$ is rotational symmetry and there is no relation between $u$ and $\varphi$. Omit partial differential item on $\varphi$ and make Laplace expansion as (2).

$$
\frac{\partial}{\partial \alpha}\left(\frac{\operatorname{sh} \alpha}{\operatorname{ch} \alpha-\cos \beta} \cdot \frac{\partial u}{\partial \alpha}\right)+\frac{\partial}{\partial \beta}\left(\frac{\operatorname{sh} \alpha}{\operatorname{ch} \alpha-\cos \beta} \cdot \frac{\partial u}{\partial \beta}\right)=0
$$

Use separation of variables to represent $u(\alpha, \beta, \varphi)=$ $\sqrt{2 \operatorname{ch} \alpha-2 \operatorname{ch} \cos \beta} X(\alpha) Y(\beta)$ and take it into equation (2), so equation (3) and (4) can be obtained, where $b$ is the coefficient.

$$
\begin{gathered}
Y(\beta)=\exp ( \pm i b \beta) \\
\frac{1}{\operatorname{sh} \alpha} \frac{\mathrm{d}}{\mathrm{d} \alpha}\left(\operatorname{sh} \alpha \frac{\mathrm{d} X}{\mathrm{~d} \alpha}\right)-\left(b^{2}-\frac{1}{4}\right) X=0
\end{gathered}
$$

Equation (4) is the Legendre equation, its solution is the Legendre function as (5) shows.

$$
X(\alpha) \sim\left\{\begin{array}{c}
P_{b-\frac{1}{2}}(\operatorname{ch} \alpha), Q_{-\frac{1}{2}}(\operatorname{ch} \alpha), b \text { is real number } \\
P_{i \tau-\frac{1}{2}}(\operatorname{ch} \alpha), Q_{i \tau-\frac{1}{2}}(\operatorname{ch} \alpha), b \text { is imaginary number }
\end{array}\right.
$$

Because the potential function is bounded, the equation (4) remains bounded and just take the first Legendre equation of (5) into account, where $P_{1}($ ch $\alpha), b=i \tau$. To get the general expression of potental function $u$ as (6) shows, take the solutions of equation (3) and (4) into it, denoting the coefficient functions of $\tau$ by $A(\tau)$ and $B(\tau)$.

$$
\begin{aligned}
u= & \sqrt{2 \operatorname{ch} \alpha-2 \operatorname{ch} \beta} \\
& \cdot \int_{0}^{\infty}\left\{[A(\tau) \operatorname{ch}(\tau \beta)+B(\tau) \operatorname{sh}(\tau \beta)] \cdot P_{i \tau-\frac{1}{2}}(\operatorname{ch} \alpha)\right\} d \tau
\end{aligned}
$$

The spherical cap meets $\beta=\beta_{0}$ and point coordinates outside the spherical cap meet $\beta_{0} \leq \beta \leq \beta+2 \pi$, and expression (6) can be adapted to (7).

$$
\begin{aligned}
u= & \sqrt{2 \operatorname{ch} \alpha-2 \operatorname{ch} \beta} . \\
& \cdot \int_{0}^{\infty} \frac{C_{1}(\tau) \operatorname{sh} \tau\left(\beta-\beta_{0}\right)+C_{2}(\tau) \operatorname{sh} \tau\left(2 \pi+\beta_{0}-\beta\right)}{\operatorname{sh}(2 \pi \tau)} \\
& \cdot P_{i \tau-\frac{1}{2}}(\operatorname{ch} \alpha) \cdot \mathrm{d} \tau
\end{aligned}
$$

According to the boundary conditions $\left.u\right|_{\beta=\beta_{0}}=$ $\left.u\right|_{\beta=\beta_{0}+2 \pi}=u_{0}$ and method of undetermined coefficients, $C_{1}(\tau)$ and $C_{2}(\tau)$ can be established by expression (8).

$$
C_{1}(\tau)=C_{2}(\tau)=u_{0} \frac{\operatorname{ch}\left(\pi-\beta_{0}\right) \tau}{\operatorname{ch}(\pi \tau)}
$$

Then with substitution Equation (7) is rewritten as

$$
\begin{aligned}
& \left.u\right|_{\beta=\beta_{0}}=u=\sqrt{2 \operatorname{ch} \alpha-2 \operatorname{ch} \beta} . \\
& \quad \cdot \int_{0}^{\infty} \frac{\operatorname{ch}\left(\pi-\beta_{0}\right) \tau \cdot \operatorname{ch}\left(\pi+\beta_{0}-\beta\right) \tau}{\operatorname{ch}^{2}(2 \pi \tau)} \cdot P_{i \tau-\frac{1}{2}}(\operatorname{ch} \alpha) \cdot \mathrm{d} \tau
\end{aligned}
$$

Use integral formula of Legendre

$$
P_{i \tau-\frac{1}{2}}(\operatorname{ch} \alpha)=\frac{2}{\pi} \operatorname{ch}(\pi \tau) \int_{0}^{\infty} \frac{\cos (\tau \theta)}{\sqrt{2 \operatorname{ch} \theta+2 \operatorname{ch} \alpha}} \mathrm{d} \theta
$$

Finally, the electric potential distribution formula (11) can be got by using Fourier integral transformation.

$$
\begin{aligned}
u=\frac{u_{0}}{2}\left\{1+\sqrt{\frac{\operatorname{ch} \alpha-\cos \beta}{\operatorname{ch} \alpha-\cos \left(2 \beta_{0}-\beta\right)}}\right. \\
+\frac{2}{\pi} \arctan \left(\frac{\sqrt{2} \cos \frac{\beta}{2}}{\sqrt{\operatorname{ch} \alpha-\cos \beta}}\right) \\
-\frac{\pi}{2} \sqrt{\frac{\operatorname{ch} \alpha-\cos \beta}{\operatorname{ch} \alpha-\cos \left(2 \beta_{0}-\beta\right)}} \\
\left.\cdot \arctan \left[\frac{\sqrt{2} \cos \left(\beta-\frac{\beta_{0}}{2}\right)}{\sqrt{\operatorname{ch} \alpha-\cos \left(2 \beta_{0}-\beta\right)}}\right]\right\}
\end{aligned}
$$

Based on the space distribution of electric potential, the capacitance of the converter can also be deduced by the following steps.

The charge distribution of the converter can be solved through the following equations. 


$$
\begin{gathered}
\sigma=\left.\varepsilon_{0} \vec{E}_{\beta} \cdot \vec{n}\right|_{\beta} \\
-\left.\varepsilon_{0} \frac{1}{h_{\beta}} \frac{\partial u}{\partial \beta}\right|_{\beta_{0}}\left(\text { internal surface }, \overrightarrow{e_{\beta}}=\vec{n}\right) \\
\left.\varepsilon_{0} \frac{1}{h_{\beta}} \frac{\partial u}{\partial \beta}\right|_{\beta_{0}+2 \pi}\left(\text { external surface }, \overrightarrow{e_{\beta}}=-\vec{n}\right) \\
\sigma_{\text {in }}=\frac{\varepsilon_{0} u_{0}}{\pi \alpha} \sin \frac{\beta_{0}}{2}\left[\sqrt{2 \operatorname{ch} \alpha-2 \cos \beta_{0}}\right. \\
\left.-2 \cos \frac{\beta_{0}}{2} \cdot \operatorname{arccot}\left(\frac{\sqrt{2} \cos \frac{\beta_{0}}{2}}{\sqrt{\operatorname{ch} \alpha-\cos \beta_{0}}}\right)\right] \\
\sigma_{\mathrm{ex}}=\sigma_{\text {in }}+\frac{\varepsilon_{0} u_{0}}{\alpha} \sin \beta_{0}
\end{gathered}
$$

where, $\sigma_{\text {in }}$ and $\sigma_{\text {ex }}$ is the density of internal and external surface charge respectively.

The charge on the internal surface of the converter $q_{\text {in }}$ is calculated as (15), the same method can be used to calculate $q_{\text {ex }}$, finally the total charge $q_{\text {all }}$ can be completed by (16).

$$
\begin{gathered}
q_{\mathrm{in}}=\iint \sigma_{\mathrm{in}} \mathrm{d} s=2 \varepsilon_{0} u_{0} a\left(1+\frac{\pi}{2} \tan \frac{\beta_{0}}{2}-\frac{\beta_{0}}{\sin \beta_{0}}\right) \\
q_{\mathrm{all}}=q_{\mathrm{in}}+q_{\mathrm{ex}}=4 \varepsilon_{0} u_{0} a \pi\left(1+\frac{\pi-\beta_{0}}{\sin \beta_{0}}\right)
\end{gathered}
$$

Here, $\delta=\pi-\beta_{0}$ and $\sin \beta_{0}=\sin \delta=a / R$, then equation (16) is rewritten as

$$
q_{\mathrm{all}}=4 \varepsilon_{0} u_{0} R(\delta+\sin \delta)
$$

The capacitance of converter is got.

$$
C_{\mathrm{sph}}=\frac{q_{\mathrm{all}}}{u_{0}}=4 \varepsilon_{0} R(\delta+\sin \delta)
$$

The capacitance formula (19) with parameters of $R$ and $a$ is rewritten by using the coordinate transformation, corresponding to the three cases in Figure 2.

$$
C_{\mathrm{sph}}= \begin{cases}4 \varepsilon_{0} R\left[\frac{a}{R}+\left(\pi-\arcsin \frac{a}{R}\right)\right] & \delta>\frac{\pi}{2} \\ 4 \varepsilon_{0} R\left(1+\frac{\pi}{2}\right) & \delta=\frac{\pi}{2} \\ 4 \varepsilon_{0} R\left[\frac{a}{R}+\arcsin \frac{a}{R}\right] & \delta<\frac{\pi}{2}\end{cases}
$$

\section{Optimization of Capacitive Converter}

In order to compare the effect of energy harvesting, define the incremental coefficient $n$ which is the ratio of the two capacitances within the same volume.

$$
n=\frac{C_{\text {sph }}}{C_{\text {flat }}}
$$

As a result, optimization of the converter can be transformed into the optimization of incremental coefficient $n$, described as equation (21).

$$
\left\{\begin{array}{c}
\max n=\frac{C_{\mathrm{sph}}}{C_{\text {flat }}} \\
\text { s.t. } V_{\mathrm{sph}} \leq V \\
V_{\text {flat }} \leq V
\end{array}\right.
$$

Its goal is to maximize the increment factor $n$, while the constraint is limited by its volume $V$. Bring the capacitance of traditional flat-plate converter and the spherical cap one into formula (20) then equation (22) can be got.

$$
n=\left\{\begin{array}{r}
\frac{4}{\pi} \cdot\left[\frac{a}{R}+\pi-\arcsin \frac{a}{R}\right] \cdot\left[1+\sqrt{\left.1-\left(\frac{a}{R}\right)^{2}\right]}\right. \\
\frac{\left(\delta>\frac{\pi}{2}\right)}{\pi\left[\frac{a}{\mathrm{R}}+\arcsin \frac{a}{R}\right] \cdot\left[1-\sqrt{1-\left(\frac{a}{R}\right)^{2}}\right]} \\
\left(\frac{a}{R}\right)^{2} \\
\left(\delta<\frac{\pi}{2}\right)
\end{array}\right.
$$

where, $\delta>90^{\circ}, V$ is limited by a cylinder with radius $R$ and height $R+a$, while $\delta<90^{\circ}, V$ is limited by a cylinder with radius $a$ and height $R-a$.

It is clear that incremental coefficient $n$ is the function of $a / R$, and it satisfies $0<a / R<1$. As a reference, Table 1 shows the calculated incremental coefficient $n$ for different $a / R$, which reflects changes of opening radius $a$ to the improvement of efficiency.

Table 1. Incremental factor for different sizes of converter.

\begin{tabular}{cccc}
\hline & $\delta>90^{\circ}$ & \multicolumn{3}{c}{$\delta<90^{\circ}$} \\
\hline$a / R$ & $n$ & $a / R$ & $n$ \\
\hline 0.1 & 9.97959 & 0.1 & 0.12775 \\
0.2 & 9.9158 & 0.2 & 0.25813 \\
0.3 & 7.8041 & 0.3 & 0.39403 \\
0.4 & 7.638 & 0.4 & 0.53913 \\
0.5 & 7.408 & 0.5 & 0.69843 \\
0.6 & 7.1003 & 0.6 & 0.8796 \\
0.7 & 6.692 & 0.7 & 1.0959 \\
0.8 & 6.1407 & 0.8 & 1.3745 \\
0.9 & 5.3418 & 0.9 & 1.791 \\
\hline
\end{tabular}


Table 1 shows that, when $\delta>90^{\circ}$, the smaller is the opening radius $a$, the more close to a ball, the greater is the capacitance, and the more energy it stores. Limited by the bottom plate grounded, the spherical cap can not be infinitely close to a ball. When $\delta<90^{\circ}$, the smaller is the opening radius $a$, the less energy it can store. When $a / R$ is 0.7 , the increment factor $\mathrm{n}$ is 1.0959 , it begins to increase the stored energy. Therefore, $a / R$ at least selected in 0.7 can improve the efficiency of energy storage (equivalent to the flat-plate converter). In particular, when $\delta=90^{\circ}$, the incremental coefficient $\mathrm{n}$ is 3.27 .

\section{Experiment about Incremental Coefficient}

To verify the validity of the analytical model, an experiment about the increment factor $n$ is done. It can get measured capacitance for different sizes of flat-plate converter and the spherical cap one by using high precision LCR meter named Quadtech1869 M. Figure 3 shows different samples of the converter with different dimensions. The height of two plates can be regulated by insulating pillars to meet the limitations of volume. Measurements and theoretical values of incremental coefficient $n$ are shown in Table 2.

The results in Table 2 show that the measured values $n$ agree well with the theoretical ones. For $\delta>90^{\circ}$, when $a / R$ is 0.5 , the error of measured values $n$ with the theoretical ones is small; but when $a / R$ increases to 0.6 , the measured values is smaller, because with $a / R$ increases,

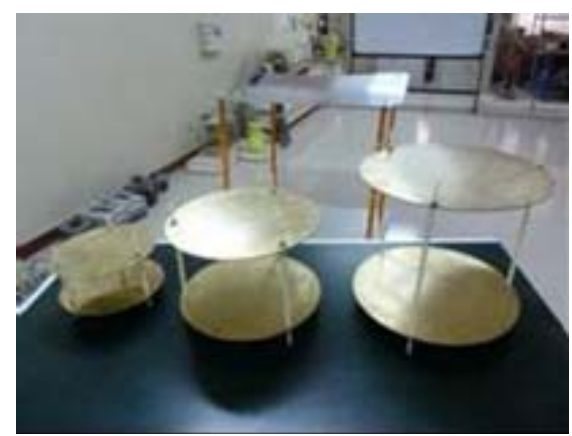

(a) Flat-plate converter

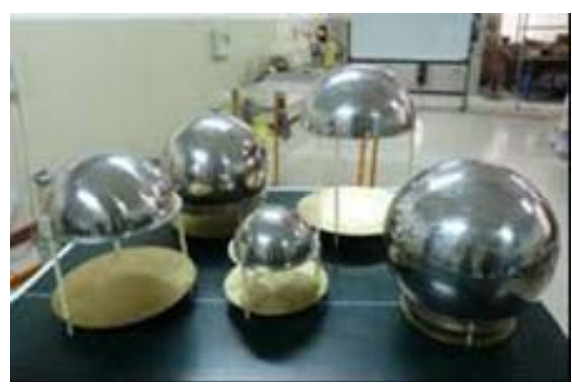

(b) Spherical cap converter

Figure 3. Different samples of the converter with different dimensions.
Table 2. Theoretical value and measured value of incremental factor.

\begin{tabular}{cccc}
\hline Types & $a / R$ & Theoretical value $n$ & Measured value $n$ \\
\hline$\delta>90^{\circ}$ & 0.5 & 7.408 & 7.0137 \\
& 0.6 & 7.1003 & 5.9472 \\
$\delta<90^{\circ}$ & 0.6 & 0.8796 & 0.7745 \\
& 0.8 & 1.3745 & 1.4628 \\
$\delta=90^{\circ}$ & 1.0 & 3.27389 & 3.15629 \\
\hline
\end{tabular}

the spherical cap is more close to a ball, the electrostatic shielding becomes stronger. For $\delta<90^{\circ}$, only when $a / R$ is greater than 0.7 , it can improve the effect of energy storage. In particular, for $\delta=90^{\circ}$ the measured value is 3.156 , and the capacitance increases three times compared with the flat-plate converter in the same volume. The experiment shows that the analytical model of spherical cap converter in the torrid coordinate system is valid.

\section{Conclusions}

This paper focuses on capacitive converter of energy scavenging system based on electric field energy to carry out research on theoretical analysis, modeling, optimization, and experiments. Analytic forms of the electric potential and capacitance is deduced based on the separated variables in the torrid coordinate system. Incremental factor about energy storage is proposed to compare the effect of energy harvesting. At last, the proposed modeling and optimization methodology are verified by testing experiments.

\section{REFERENCES}

[1] Y. X. Yu and W. P. Luan, "Smart Grid and Its Implementations," Proceedings of the CSEE, Vol. 29, No. 34, 2009, pp. 1-8.

[2] A. P. Joseph and S. Thad, "Energy Scavenging for Mobile and Wireless Electronics", IEEE Transactions on Pervasive Computing, Vol. 4, No. 1, 2005, pp. 18-27.

\section{doi:10.1109/MPRV.2005.9}

[3] D. S. Lee, "Wireless and Powerless Sensing Node System Developed for Monitoring Motors," Sensors, Vol. 8, No. 8, 2008, pp. 5005-5022. doi:10.3390/s8085005

[4] Y. G. Sun, "Study on Structure Vibration Wireless Sensor Based on Solar Cell," Noise and Vibration Control, No. 4, 2007, pp. 132-133.

[5] S. S. Nathan and A. P. Joseph, "Energy Scavenging with Shoe-mounted Piezoelectrics," IEEE Micro, Vol. 21, No. 3, 2001, pp. 30-42.

[6] M. Zhu, P. C. Baker and N. M. Roscoe, "Alternative Power Sources for Autonomous Sensors in High Voltage Plant," 2009 IEEE Electrical Insulation Conference, 
Montreal, Canada, May 31-June 3, 2009, pp. 36-40

[7] X. Li, "Computation of the Eccentric Spherical Capacitor's Capacitance," Journal of Capital Normal
University Natural Sciences Edition, Vol. 24, No. 4, 2003, pp. 34-35. 